\title{
A ATUAÇÃO DO DESIGNER DE SUPERFÍCIE E OS CONDICIONANTES CULTURAIS DIANTE DO CENÁRIO DA CÓPIA
}

\author{
Larissa Fernanda de Barros Mota, mestranda \\ Universidade Federal de Pernambuco \\ larissa.barros1987@gmail.com \\ Kátia Medeiros de Araújo, Dra. \\ Universidade Federal de Pernambuco \\ katia_araujo@hotmail.com
}

Resumo: Este estudo propõe uma discussão acerca do papel do designer na sociedade diante do contexto cultural em que ele se insere. Nesta perspectiva, atentamo-nos para a cultura da cópia presente na Cidade de Caruaru-PE, a qual faz parte do Polo de Confecções do Agreste Pernambucano, apresentando um faturamento considerável. Nesse contexto, foram realizadas entrevistas semiabertas, de base qualitativa, a designers de superfície têxtil atuantes na região. Para fomentar a discussão acerca do tema, foram realizadas pesquisas bibliográficas sobre design e território, cultura da cópia e seus reflexos na atuação do designer e desafios contemporâneos. Por fim, são apresentados e discutidos os impactos e representações que a cultura da cópia causa à atuação do designer na região.

Palavras-chave: Design de Superfície; Cultura da Cópia, Pólo de Confecções do Agreste-PE.

\begin{abstract}
This study proposes a discussion about the designer's social role, taking into consideration the cultural context in which these professional are inserted. In this perspective, we emphasize the culture of copy in the city of Caruaru-PE, which is part of the Pólo de Confecções do Agreste Pernambucano, that presents a considerable volume of business. In this context, we applied semi-open interviews constructed in qualitative basis to textile designers that work in the region. To encourage discussion on the subject, were conducted literature searches about design and territory, culture of copy and its impacts on the work of the designers and contemporary challenges. Finally, are presented and discussed the impacts and representations of the culture of copy to the role of the designer in the region.
\end{abstract}

Keywords: Surface Design; Culture of Copy, Clothing Design in Caruaru Pernambuco State 


\section{INTRODUÇÃO}

Atualmente, o design tem se revelado um fator de grande importância e responsabilidade para o desenvolvimento do capital e humano das sociedades. Compreendendo este cenário, podemos perceber que o design tem muito a contribuir no contexto globalizado, por ser uma atividade que acompanha a inovação e se volta para a inserção de produtos tecnológicos e inovadores para compor o sistema produtivo.

[...] para países produtores e designers que atuam em cenários mutantes, fluidos e complexos, o desafio deixa de ser o âmbito tecnicista e linear, passando à arena ainda pouco conhecida e decodificada dos atributos intangíveis dos bens de produção industrial. Isso faz também com que o design interaja, de forma transversal, com disciplinas cada vez menos objetivas e exatas, passando a confluir com outras que compõem o comportamento humano e social, os fatores sensoriais e psicológicos (MORAES, 2010, p. 14).

Com estes novos cenários do mundo globalizado, a proposta é refletir sobre o contexto dessas mudanças e sua interferência sobre a prática do design, uma vez que reconhecemos nessa atividade, tal com o faz Bonsiepe (2012) e outros autores, um poder de ferramenta estratégica diante das necessidades em âmbito local. Deste modo, é importante voltar-se para o cenário local como estratégia de diferenciação, reconhecendo e interpretando os códigos que constituem o território.

Nesse sentido, faz-se necessário o designer ser capaz de interpretar as demandas locais, perceber os costumes e fenômenos pertencentes à região em que atua, conforme ratifica Moraes:

[...] torna-se imperativa a capacidade que produtores, designers e mesmo o país tem de interpretar o estilo de vida local (local culture e local lifestyle) para que ele seja inserido como componente diferencial dos produtos que competem hoje em nível global. (Moraes, 2010, p.14).

Objetivando promover discussões acerca do papel do designer no desenvolvimento local de determinado contexto, este artigo busca refletir sobre as características de atuação do designer de superfície empregadas atualmente, tendo como principal foco a cultura da cópia evidenciada no contexto do Pólo de Confecções do Agreste - Pernambuco/BR. Assim, tencionamos discutir acerca de como ela pode interferir nos processos de criação do designer.

Neste estudo, de aspecto exploratório e base qualitativa, foram realizados levantamento de dados bibliográficos e documentais acerca das temáticas que envolvem a prática da cópia, as teorias de Imitação e Diferenciação em Moda, Design e Território e, brevemente, uma explanação acerca do design de superfície (termos e definições). Além disso, foram realizadas entrevistas semiestruturadas com designers de estamparia têxtil, residentes e atuantes na cidade de Caruaru, como estratégia de aproximação e compreensão da citada problemática neste universo específico.

A motivação que nos levou ao estudo reside no anseio por explorar de maneira diferenciada e minuciosa os fenômenos sociais e culturais da região, processo que se configura como uma rica ferramenta de concepção e criação de design, mas permanece pouco explorado no Pólo de Confecções do Agreste de Pernambuco. 
Assim, objetivamos considerar a atividade projetual dos designers da região para além da busca de referências a partir de tendências internacionais de moda, o que se tornou prática recorrente entre os profissionais que atuam no citado contexto. Por entendermos que o caminho para transformações renovadoras, em quaisquer que sejam os campos de práticas, se inicia pelo entendimento aprofundado das problemáticas envolvidas com tais práticas, objetivamos com esse estudo, proporcionar, mais especificamente, maior entendimento sobre o comportamento dos profissionais que atuam no design de estamparias no contexto do local.

\title{
2 DESENVOLVIMENTO
}

Com a intenção de inserir a discussão acerca da importância dos códigos revelados da cultura local, a pesquisa se volta para a APL da cidade de Caruaru, no estado de Pernambuco, com o objetivo de favorecer um dos polos de confecção de maior importância econômica e de produção para a região.

A fundamentação teórica corresponde aos temas principais em questão, que auxiliarão na discussão em tela.

\subsection{Design de superfície têxtil}

Temos registros de elementos gráficos sobre as superfícies desde o paleolítico. As civilizações antigas utilizavam figuras com tendência à repetição de motivos como forma de decoração, inclusive na elaboração do vestuário, conforme mencionado abaixo:

\begin{abstract}
As civilizações antigas desenvolveram o gosto pela decoração de superfícies em geral, principalmente em utensílios domésticos, espaços arquitetônicos e artefatos têxteis. Pode-se dizer que a tecelagem e a cerâmica, assim como, posteriormente, a estampa e a azulejaria, com sua linguagem visual, carregam o embrião do que hoje chamamos de design de superfície (RÜTHSCHILLING, 2008, p. 16).
\end{abstract}

O Design de Superfície, sendo uma modalidade relativamente recente no Brasil, abrange um leque de possibilidades e variações, tais como a aplicação de superfície em cerâmica, tecidos, louça, entre outros (RUBIM, 2005).

A característica principal da representação do Design de superfície é a ideia de repetição do desenho. A respeito do tema, Rubim esclarece:

Para se referir a essa forma de representação - um desenho em repetição, modulado -, se utiliza, na grande maioria das indústrias brasileiras o termo rapport, originário do francês. A denominação em inglês é repeat. (RUBIM, 2005, p. 36).

Vale ressaltar, que, no processo de desenvolvimento, criação ou seleção de estampas para compor um produto de moda, além de buscar referências de informações de moda nas fontes globais diversas, o designer deve conhecer o mercado em que irá atuar compreendendo o seu público e as adequações ao mercado. BriggsGoode salienta a importância de entender o publico alvo e acrescenta ser essencial se conhecer as exigências do mercado, devendo-se "[...] considerar como isso afeta a sua 
interpretação ou o uso de imagem, cor, materiais, legado da estampa e, principalmente, seu processo de criação." (BRIGGS-GOODE, 2014, p. 31).

\subsection{Design e território}

Lia Krucken, em seu livro Design e Território, promove uma reflexão acerca da valorização dos produtos locais. Para a autora, estes produtos revelam as manifestações culturais que pertencem ao local de onde eles foram gerados, bem como os próprios costumes e hábitos da região. Nessa mesma perspectiva, a autora discorre acerca do termo terroir, de modo que "[...] abrange o produto, o território e a sociedade que o produz [...]" (KRUCKEN, 2009, p. 31). Sendo, neste caso, um aspecto que caracteriza-se pela interação do homem e o contexto local.

$\mathrm{O}$ design desempenha um papel de mediador entre as dimensões imateriais e as dimensões materiais envolvidas nos projetos. Há, na contemporaneidade, uma evidente dinâmica de acontecimentos que se refletem em aspectos culturais, econômicos e sociais. Nesse contexto, a autora aborda o design como uma tendência de configurar os aspectos socioculturais e tecnológicos como um vetor para a competitividade. "[...] a conscientização de profissionais - industriais, empresários, pesquisadores - da importância de se investir em design vem se consolidando nos últimos anos." (KRUCKEN, 2009, p.43). Em um cenário globalizado, a complexidade do design se apresenta no desafio de encontrar oportunidades numa relação que sutilmente se coloca entre o global e o local.

A busca por coerência na concepção dos artefatos, com vistas às inúmeras dinâmicas sociais e culturais, deve fazer parte da abordagem do design, reforçando assim o seu caráter sistêmico e levando em consideração a cultura que envolve os objetos. Por sua vez, os produtos que são reveladores das manifestações culturais locais estão fortemente ligados ao território.

Reconhecer esse território, além de compreender os fenômenos que o envolvem caracteriza uma preocupação necessária para que se projete a partir de conteúdos condizentes. "Assim, para dinamizar os recursos do território e valorizar seu patrimônio cultural imaterial, é fundamental reconhecer e tornar reconhecíveis valores e qualidades locais. Essa é uma das principais tarefas do designer." (KRUCKEN, 2009, p. 18).

Krucken (2009) descreve três características em que o designer pode contribuir para a valorização dos produtos locais; são elas: promover a qualidade do produto, apoiar a comunicação, intensificando as relações territoriais e apoiar os arranjos produtivos e cadeias de valor.

Desta forma, promover esta visibilidade e buscar condições para o desenvolvimento potencial a partir das possibilidades dos recursos locais para que estes possam trazer benefícios para a sociedade e um melhor desenvolvimento constitui uma tarefa continua do cenário globalizado. O design, enquanto campo de práticas de projeto deve, portanto, ser capaz de promover soluções, favorecendo as potencialidades locais e promovendo a integração entre o "global" e o "local".

$O$ design pode facilitar o processo de inovação em um desenvolvimento econômico e sociocultural, nos diversos sentidos. Mas, principalmente, é necessário se conhecer, a partir de uma visão panorâmica e sistêmica, os aspectos territoriais para que se possa elaborar um projeto de valorização local, sob a perspectiva do design. Existem algumas ações necessárias neste eixo: Reconhecer as qualidades, ativar as 
competências do território, comunicar o produto e território, proteger a identidade local, apoiar a produção local, promover sistemas de produção e consumo sustentáveis, desenvolver novos produtos que respeitem e valorizem o território e consolidar redes no território. (KRUCKEN, 2009, p. 99).

\title{
2.3 Cultura da cópia
}

Antes de tudo, é necessário apontar os diversos sentidos acerca do termo "cópia". Primeiramente, a definição do dicionário demonstra algumas das diversas possibilidades e significados sobre o mesmo tema. Senão vejamos:

\begin{abstract}
sf (lat copia) 1 Reprodução textual do que está escrito algures; transcrito, traslado. 2 Imitação, transcrito, calco ou reprodução de uma obra original, tal como escrito ou impresso, gravura, pintura, estátua, vestido, móvel, filme cinematográfico etc. 3 Reprodução fotográfica. 4 Reprodução de uma escrita datilografada, produzida mediante papel-carbono. $\mathbf{5}$ Imitação, plágio. 6 Pessoa ou coisa semelhante a outra. 7 Abundância, grande número, grande quantidade.[...] (MICHAELIS, 2016).
\end{abstract}

Fazendo um paralelo da cultura da cópia com a teoria da Periferia e Centro proposta por Gui Bonsiepe, observamos ser a base principal dos processos de cópia a falta de apropriação da inovação que é possível a diferentes ambientes tecnológicos e culturais. O fenômeno é apontado como um grave problema da Periferia, enquanto que nas culturas tecnológicas dos países desenvolvidos (centrais) haveria uma consciência mais precisa sobre as possibilidades de inovação, ou melhor, de apropriação dos produtos realizados pela ciência em forma de tecnologia, inovação social, etc. Ainda segundo as formulações do autor, a consciência dos países centrais em relação ao poder conferido pela capacidade de inovação se estende à noção exata da dominação que podem exercer sobre países periféricos, a partir da comercialização dos produtos tecnológicos (ou do próprio conhecimento tecnológico e industrial), de forma assimétrica e à revelia dos interesses econômicos, sociais e das disposições culturais dos compradores dessas tecnologias.

O autor discorre sobre as possíveis soluções para essa discrepância entre países Centrais e Periféricos: "Só haverá mudança do estado de dependência, passando para interdependência, quando a industrialização nos países dependentes for acompanhada pela inovação tecnológica para abastecer a indústria local" (BONSIEPE, 2012, p. 41).

Para Gui Bonsiepe, a definição de Periferia e Centro diz respeito principalmente a uma divisão de atribuições em nível internacional, onde os produtos "commodities", como, por exemplo, os recursos naturais não transformados, são a parte exportada pela Periferia e, por outro lado, os países do Centro exportam tecnologia, vendendo para as periferias, muitas vezes, conhecimentos tecnológicos superados e nada inovadores.

Como sugerido pelo autor, sabe-se que diversos princípios do design utilizados no contexto Brasileiro, relativos aos conteúdos de tendências e predefinições macro, são advindos do Centro. Erroneamente, a Periferia adquire o papel de mimetizar, ou seja, de copiar as ações do Centro, replicando o seu processo.

A imitação é um fenômeno intrínseco ao processo da moda. Isso caracteriza a face de um sistema dualista de comportamento social, onde se apresenta, por um 
lado, a imitação, trazendo o indivíduo para um sistema coletivo; e por outro, a diferenciação, que se dá basicamente pela negação do ato de imitar.

O fenômeno da imitação se apresenta, portanto, como uma importante forma de comportamento, passível de observação com destaque pelas sociedades como um todo.

Ela opera uma espécie de "passagem da vida do grupo na vida individual", facultando ao indivíduo assimilar-se em meio ao grupo, como parte dele, como um "recipiente de conteúdos sociais". O princípio de imitação representa assim um lado do dualismo, cuja outra face é dada pela diferenciação individual, pela negação do imitar - o inventar. A imitação é "uma das direções básicas de nossa essência" e possibilita a "fusão do singular na universalidade", ou seja, "enfatiza em meio à mudança aquilo que permanece". (WAIZBORT, 2008, p. 9).

Carvalho e Pirauá (2008), em um estudo relacionado ao fenômeno da Imitação na cidade de Toritama e sua confecção de jeans- um produto de grande produção na região -, afirmam que, sendo a imitação um comportamento geral de repetição de modelos, esta característica "[...] tem grande relevância para a moda, pois permite uma identificação, através do vestuário, com as tendências internacionais. Fenômeno característico do pólo de confecções do Agreste, a imitação, torna-se um meio de segurança e de sucesso em vendas [...]" (CARVALHO e PIRAUÁ, 2008, p. 326).

\subsubsection{Contexto local - Caruaru-PE}

As cidades de Caruaru, Toritama e Santa Cruz do Capibaribe formam um grande centro de confeç̧ões do agreste pernambucano. Conhecidos por sua força devida à grande produção, estas cidades formam o triângulo de base, considerado como um dos maiores produtores de confecção em moda no Brasil; senão vejamos:

O triângulo Caruaru, Santa Cruz do Capibaribe e Toritama representa 77\% em relação ao total de postos de trabalho do polo, que produz anualmente mais de 842 milhões de peças, em sua maioria artigos de consumo popular, cujos destinos são, principalmente, as regiões nordeste e sudeste, embora a Rodada de Negócios da Moda Pernambucana registre vendas para todos os estados brasileiros. (RODADA DE MODA DE PERNAMBUCO, 2016).

Esse estudo engloba os fenômenos culturais e possíveis limitadores no contexto da cópia do APL da cidade de Caruaru-PE, a qual faz parte do Polo de Confecções do Agreste pernambucano. Os APL's (Arranjos Produtivos Locais) são:

[...] uma aglomeração de empresas, localizadas em um mesmo território, que apresentam especialização produtiva e mantêm vínculos de articulação, interação, cooperação e aprendizagem entre si e com outros atores locais, tais como: governo, associações empresariais, instituições de crédito, ensino e pesquisa. (SEBRAE, 2016).

Assim, a cidade é caracterizada por sua forte economia voltada para a indústria e comercio de confecções, com incentivos governamentais e apoio voltado para os micro e pequenos empresários.

Segundo dados fornecidos pelo SEBRAE (Serviço Brasileiro de Apoio às micro e pequenas Empresas), o Polo de Confecções cresceu, em termos demográficos, 2,2 vezes mais rápido que o Brasil e 2,4 vezes mais rápido que o Nordeste, demonstrando 
assim uma migração de moradores de outros locais para este polo devido às oportunidades econômicas, demonstrando assim a clara importância de estudos e pesquisas relacionadas a este setor.

Um dado fornecido pelo Jornal do Comércio de Pernambuco revela que o "mix" de produtos são distribuídos por "[...] itens populares a artigos mais trabalhados, com $75 \%$ das 850 milhões de peças comercializadas no Nordeste e pouco mais de $12 \%$ na Região Sudeste, com destaque para Minas Gerais e Espírito Santo." (JC ONLINE, 2016).

Ainda segundo o JC Online, o Polo de Confeç̧ões se caracteriza na categoria fest fashion, ou seja, a moda rápida. Afirmando que "o que saiu na TV" rapidamente está na prateleira.

\begin{abstract}
A tendência que aparece hoje nas novelas pode ser rapidamente reproduzida. Há empresas com produção quinzenal de coleções. É aí que os negócios conseguem concorrer com a China, de onde os produtos demoram até chegar ao Brasil. Por outro lado, as peças asiáticas muitas vezes entram no País ilegalmente e terminam ficando mais baratas. Os maiores compradores do Polo continuam sendo sacoleiros e pequenos e médios lojistas. Por lá, ninguém acha muito vantajoso fechar negócio com grandes magazines, que pedem exclusividade e não dão garantia de renovação de contrato. (JC ONLINE, 2016)
\end{abstract}

Segundo levantamento feito pela Federação do Comércio de Pernambuco (Fecomércio-PE), a maioria dos compradores são os próprios moradores da região, e consomem para as necessidades próprias ou dos seus familiares. "Essa proporção é mais elevada no Polo Caruaru, onde $95,3 \%$ dos compradores adquirem os produtos para consumir." (FECOMERCIO, 2016). A feira de Caruaru, além de representar um dos principais pontos turísticos da cidade, é uma das feiras mais importantes do Estado. “[...] E para que as tradições da Feira sejam preservadas, a 5a Superintendência Regional do Iphan encaminhou um projeto para o seu registro como patrimônio imaterial, aprovado no dia 7 de dezembro." (PORTAL IPHAN, 2016).

\title{
2.3.2 Resultados e discussão
}

Por meio de entrevistas e observação de campo, foram colhidas informações relacionadas ao processo criativo dos designers, suas formações, habilidades, recursos utilizados na criação de estampas, bem como questionamentos acerca das demandas exigidas e o mercado em que estes designers atuam.

A coleta de dados, caracterizada numa fase de pré-teste, foi realizada com uma entrevista semiestruturada a 03 designers que trabalham em uma mesma empresa. $\mathrm{Na}$ ocasião, os designers foram entrevistados isoladamente, e foram utilizados recursos de gravador de voz para transcrever posteriormente. Cada entrevista durou, em média 30 minutos.

Nesse contexto, foram consideradas as informações colhidas entre os profissionais, cujas entrevistas integram os estudos piloto de uma pesquisa mais ampla. Esses profissionais atuam na criação de desenhos para estamparia rotativa no segmento de moda feminina em uma empresa na cidade de Caruaru/Pernambuco. Os entrevistados tem formação de Bacharel em Design pela Instituição Federal de ensino da própria cidade (UFPE- Campus do Agreste) recebendo em média a remuneração de $\mathrm{R} \$ 1.600,00$ a $\mathrm{R} \$ 2.300,00$, com tempo de trabalho de, respectivamente, 6 anos e 5 meses em relação ao primeiro entrevistado, 5 anos com o segundo entrevistado e 1 
ano e 9 meses, com o terceiro entrevistado, e classificações de profissional de acordo com a experiência e tempo do designer (Design Júnior, Design Pleno e Design Sênior).

Em síntese, no que tange ao processo de criação das estampas, houve convergência nas respostas no sentido de assinalarem o expressivo uso de recursos de imagens e vetores (de sites pagos e gratuitos) no processo projetual, (criativo), de modo a tornar o processo de criação mais ágil. Entre os recursos disponibilizados, foram citados os bancos de imagens livres (Freepik.com, deviantART.com), bem como portais pagos (WGSN, Usefashion.com), tanto para a busca de elementos para compor as estampas, como para obter informações de moda e tendência.

As estampas são desenvolvidas muitas vezes através de briefings, repassados pelos coordenadores de moda nos ambientes em que os designers atuam, ou por clientes específicos.

Os recursos mais utilizados são os Softwares gráficos (Photoshop e Illustrator Adobe - para criação dos elementos) e o pacote Fashion Studio da NedGraphics, especificamente os softwares Design and Repeat, Color Reduction And Cleaning e Easy Coloring (com suas funções respectivas para montagem do rapport, captura e redução de cores e coloração).

Em todas as experiências dos designers relatadas, registra-se que houve momentos em que já realizaram alguma cópia de uma marca global, como solicitação da empresa/cliente em que prestam o serviço. Esta prática é comum e bastante repetida na região.

Observou-se o uso de softwares gráficos na composição das estampas, bem como o uso de mesa digitalizadora para ajustar e criar elementos novos digitalmente. Foram citados como componentes importantes no processo de criação, além dos softwares específicos para criação de estampas (do pacote de softwares da Nedgraphics), os recursos relacionados à fotografia de texturas diferenciadas, bem como os desenhos e pinturas feitos à mão, que foram mencionados como forma de se obter composição autoral dos desenhos. Porém, os entrevistados foram unânimes em afirmar que não há possibilidade de utilizar estes recursos devido ao fator emergencial na criação. Criar a partir de texturas criadas pelos designers, por fotografias próprias ou até mesmo pela utilização de desenho à mão livre e pintura requer um tempo não disponível em seus processos de trabalho, o que inviabiliza a prática dessas ações.

Neste caso, o processo de criação se caracteriza pela busca dos elementos (figuras e imagens já existentes na internet) e manipulação dos mesmos a partir dos softwares gráficos, demonstrando-se uma prática recorrente no dia a dia desses designers. Contudo, vale salientar que não houve manifestação de satisfações dos profissionais que trabalham com o design de estamparia no contexto do citado Pólo de Confecções do Agreste, diante desta prática.

Diante da conscientização dos designers e da observação realizada, nota-se que na região há uma insistência em associar exclusividade e inovação aos produtos de estamparias de "grandes marcas", inclusive aquelas locais que mais se destacam em termos de crescimento, observando-se também, neste ponto, a questão do baixo investimento em desenvolvimento e pesquisa dos produtos, por parte do empresariado e dos confeccionistas da região. Foi mencionado nas entrevistas que os empresários, não só admitem, como privilegiam a possibilidade de copiar produtos que já foram projetados, comercializados e que já receberam feedback positivo do mercado, em detrimento de criações originais para suas empresas, sob a justificativa 
de que consistem em processos mais baratos, além de mais seguro, por, certamente haver aceitação por parte da região.

Outro aspecto apontado é a inserção dos produtos importados na região. Nesse contexto, são trazidos produtos estampados, que chegam aos confeccionistas com preços muitas vezes mais baixos que os produzidos pelas empresas de estamparia na região. Há diferença entre os tecidos importados que já sejam fornecidos com estampas prontas e os produtos das estamparias locais, uma vez que esses últimos apresentam ainda alguma diversificação, mesmo tendo por base o processo de cópia. Apesar de trabalharem com tecidos mais baratos, as empresas que trabalham com estamparias importadas lidam, porém, com a possibilidade de utilizarem padrões já utilizados por diversas empresas, visto que os tecidos que são trazidos já estampados podem ser vendidos por diversos fornecedores, por valores que interferem na concorrência territorial na cidade.

\section{CONCLUSÃO}

Por meio de observação de campo e entrevistas, foi possível notar a atuação do designer e suas atribuições no que tange ao fenômeno da cultura da cópia evidente na região, refletindo, inclusive, acerca da emergência no processo de criação das estampas.

A partir do material coletado, pudemos perceber que a cultura da cópia inserida na cidade se caracteriza também como forma de manter seguro o lucro do que já foi consolidado no mercado, visto que o comércio caruaruense ainda tem um forte diferencial competitivo voltado para o preço.

Além da competitividade voltada quase que exclusivamente para o preço do produto, há um aspecto agravante relacionado aos produtos chineses que são inseridos na Feira de Caruaru: a comercialização dos produtos importados, devido ao baixo custo dos mesmos, potencializa o desafio do comércio dos produtos cujas estampas foram concebidas localmente (e confeccionados localmente) na cidade de Caruaru e demais cidades do Pólo, cujas feiras estão sempre inundadas por produtos importados. A concorrência do produto importado faz diminuir a qualidade e a possibilidade de viabilizar a inserção do processo de design aos produtos comercializados, promovendo um ambiente propício à opção pela cópia mais barata.

Exige-se uma produção rápida e de alta rotatividade por parte dos designers que atuam nesta área na região, e isso os impede de criar um desenho totalmente autoral.

Além da consequência negativa relativa à exploração indevida dos próprios padrões de estamparia e do esforço laboral dos designers em adaptá-las (ou copiá-las em novos arranjos) em um ritmo acelerado, observou-se que a cultura da cópia instalada na região traz diversas outras consequências negativas; entre elas: a possibilidade de serem concebidas composições de estampas muito parecidas pelos designers que atuam na região (já que se utiliza de recursos públicos, vistos e utilizados por quem quiser); a questão dos direitos autorais, que não são discutidos nem postos em prática, tanto por parte destes designers quanto pelas empresas; e finalmente, os prejuízos simbólicos para o comercio na região, e para a qualidade do produto como artefato revelador de um produto de design e identidade, já que não se privilegia a originalidade do design, mas, ao contrário, a adaptação no processo de cópia. 
A partir desta análise acerca dos problemas encontrados no contexto em questão, e com a proposta de trazer as reflexões acerca do território e identidade discutidos por Lia Krucken e Gui Bonsiepe respectivamente, percebemos a necessidade de refletir acerca da cultura da cópia vigente, bem como buscar melhorias neste cenário, visto que o designer pode ser um importante mediador na possibilidade de promover e valorizar recursos territoriais e colaborar no fomento do desenvolvimento local.

\section{REFERÊNCIAS}

BONSIEPE, Gui. Design como prática de projeto. São Paulo: Blucher, 2012.

BRIGGS-GOODE, Amanda. Design de estamparia têxtil. Porto Alegre: Bookman, 2014.

CARVALHO, Mario de; PIRAUÁ, José. O imaginário da imitação em Toritama. Revista Famecos. Porto Alegre. v. 17. n. 3. p. 324-329. setembro/dezembro. 2010.

FECOMÉRCIO. Federação do Comércio de Bens, Serviços e Turismo do Estado de

Pernambuco. Disponível na internet por http em: <http://www.fecomerciope.com.br/fecomercio/4358-compradores.html. Acesso em 03 maio 2016.

JC Online. Jornal do Commércio. Disponível na internet por http em: <http://jconline.ne10.uol.com.br/canal/economia/pernambuco/noticia/2014/12/14/p olo-de-confeccoes-do-agreste-da-sulanca-a-industrializacao-160371.php>. Acesso em 03 maio 2016.

KRUCKEN, Lia. Design e Território: valorização de identidades e produtos locais. São Paulo: Studio Nobel, 2009.

MICHAELIS, Dicionário de Português Online. Disponível na internet por http em: <http://michaelis.uol.com.br/moderno/portugues/definicao/copia\%20_934593.html>. Acesso em 03 maio 2016.

MORAES, Dijon de; KRUCKEN, Lia; REYES, Paulo. Cadernos de Estudos Avançados: identidade. Universidade do Estado de Minas Gerais. Barbacena: EdUEMG, 2010.

PORTAL IPHAN. Iphan registra Feira de Caruaru como patrimônio imaterial.

Disponível na internet por http em: http://portal.iphan.gov.br/pagina/detalhes/61. Acesso em 09 de maio 2016.

RODADA DE MODA DE PERNAMBUCO. Polo de Confecções do Agreste. Disponível na internet por http em: <http://www.rodadamodape.com/polo-de-confeccoes-doagreste-\%E2\%80\%93-arranjo-produtivo-local-impulsiona-economia-da-regiao/>. Acesso em 03 maio 2016.

RUBIM, Renata. Desenhando a superfície. São Paulo: Edições Rosari, 2004.

RÜTHSCHILLING, Evelise Anicet. Design de Superfície. Porto Alegre: Ed. UFRGS, 2008.

SEBRAE. Arranjo Produtivo Local - Série Empreendimentos Coletivos. Disponível na internet por http em: http://www.sebrae.com.br/sites/PortalSebrae/bis/arranjoprodutivo-local-serie-empreendimentoscoletivos,5980ce6326c0a410VgnVCM1000003b74010aRCRD. Acesso em 08 de maio 2016. 
WAIZBORT, LEOPOLDO. Georg Simmel sobre a moda: uma aula. IARA - Revista de Moda, Cultura e Arte - São Paulo - v.1 n. 1 abr./ago. 2008. 